\title{
Spontaneous Rupture of the Non Gravid Uterus: A Case Report
}

\author{
Saima Najam¹, Sana Abady Mohmed², Shehla Aqeel ${ }^{3}$, Ghada Abujamea ${ }^{4}$ \\ ${ }^{1}$ FCPS, PG Certification in Medical Education (Dundee), Head of the OBGYN Department, \\ Dr. Sulaiman Al Habib Hospital, Sweidi, Riyadh, Saudi Arabia, \\ ${ }^{2}$ Consultant, OBGYN, Dr. Sulaiman Al Habib Hospital, Sweidi, Riyadh, Saudi Arabia \\ ${ }^{3}$ Assistant Professor, Fatima Memorial Hospital, Lahore, Pakistan \\ ${ }^{4}$ Resident, OBGYN, Dr. Sulaiman Alhabib Hospital, Sweidi, Riyadh Saudia Arabia
}

Corresponding Author: Saima Najam

\begin{abstract}
Uterine rupture can cause serious morbidity and mortality to the women. A complete uterine rupture is a diagnosis made when all three layers of the uterus are separated, while uterine dehiscence is a similar condition in which there is incomplete division of the uterus that does not penetrate all three layers of the uterus. Uterine dehiscence is most often an occult finding in asymptomatic patients. Mostly the uterine rupture is seen in gravid females however it has been reported in non gravid patients as well. Spontaneous uterine rupture is extremely rare to be seen in non gravid patients, but should be included in the differential diagnosis of acute abdomen and shock in a non pregnant woman of any age due to its catastrophic consequences. In non gravid uterus the most common cause of the uterine rupture is pelvic trauma, uterine myomas, infection or uterine carcinoma.

We hereby report a case of 44 years old female with previous 4 caesarean sections who came on 12 th day of her cycle with heavy bleeding, lower abdominal pain and anaemia. She was found to have small fibroids which were blamed for the menorrhagia and she was admitted for the conservative management. She collapsed on day 2 of admission suddenly and after stabilization of the patient her exploratory laparotomy was done and uterine rupture was detected on the right side of the previous scar. The uterus was repaired as the patient refused for hysterectomy. Her post op recovery was uneventful.
\end{abstract}

Her first periods after the surgery was normal with average amount of blood loss.

Key Words: Uterine rupture, haemorrhage, non -gravid, caesarean, hysterectomy, laparotomy

\section{INTRODUCTION}

A complete uterine rupture is a diagnosis made when all three layers of the uterus are separated, while uterine dehiscence is a similar condition in which there is incomplete division of the uterus that does not penetrate all three layers of the uterus. Uterine dehiscence is most often an occult finding in asymptomatic patients.(1) most uterine ruptures occur in gravid females however it has been reported in non gravid patients as well. (2) Uterine rupture can cause serious morbidity and mortality to the women. Spontaneous uterine rupture is extremely rare to be seen in non gravid patients, but should be included in the differential diagnosis of acute abdomen and shock in a non pregnant women of any age due to its catastrophic consequences.(2) In non gravid uterus the most common cause of the uterine rupture is pelvic trauma, uterine myomas, infection or uterine carcinoma.(3)

\section{CASE REPORT}

We are reporting a case of 44 years old Middle Eastern women who was para 5 and had one abortion. She had history of 
previous 4 caesarean sections preceded by the first spontaneous vertex delivery. Her last child born was 11 years ago. Her last pregnancy was 2 year ago in which she presented at 5 weeks and 5 days with missed abortion and bleeding for which evacuation and curettage was done.

She presented on the $12^{\text {th }}$ day of her cycle in emergency with excessive vaginal bleeding from last 12 days and right iliac fossa pain from last 12 hours. The bleeding was moderate and the pain was dull aching, which aggravated on movement. She denied any history of contraception, trauma, fever, dysuria, vomiting and constipation. She was non smoker and non alcoholic. She looked distressed and had dyspnea with little tachycardia and the blood pressure was normal. On abdominal examination she had mild suprapubic tenderness. Her BHCG (BHuman Chorionic Gonadotropin) was less than $1.20 \mathrm{mIU} / \mathrm{ml}$. (milli international units per milli liters), showing non pregnant levels. Her CBC (complete blood count) showed normal WBC (white blood cells count), with HGB (Haemoglobin) 8.1 gm/dl.(grams per deciliters) and HCT (hematocrat) $27.2 \%$. With normal platelets. Her ultrasonography of the abdomen and pelvis, was unremarkable except that she was found to have multiple fibroids largest being $3 * 2.7 \mathrm{~cm}$ in the fundal region. Her TVS (trans-vaginal ultrasonography) confirmed the fibroids, and the fluid collection was seen at the site of the uterine scar, as shown in figure 1.

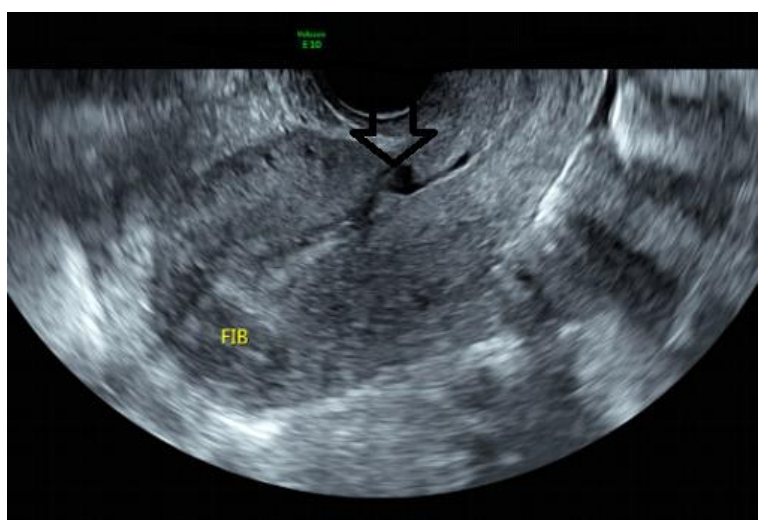

FIGURE 1: Transvaginal ultrasonography, showing multiple fibroids and the suspicious fluid at the area of the scar
Her urine analysis was normal. The patient was admitted for monitoring, blood transfusion as she was symptomatic and for the injectable anti fibrinolytic therapy with NSAID (non steroidal anti inflammatory drugs) and progesterone.

The patient started responding to the treatment and the bleeding reduced, till on the second day of the admission when she complained of sudden onset of dizziness and heavy episode of bleeding. Her pulse was 110 beats per minutes, her blood pressure was $81 / 42 \mathrm{~mm}$ of $\mathrm{Hg}$ (millimeters of mercury) and she was afebrile. Her abdomen was soft except the suprapubic tenderness noted earlier. RRT (rapid response team) was activated and the patient was shifted to ICU (intensive care unit) Where she was stabilized and on repeat investigations the HGB was $7.0 \mathrm{gm} / \mathrm{dl}$ after one unit of the PRBC.(packed red blood cells). The patient was counselled for exploratory laparotomy and hysterectomy, but she refused and signed the AMA (against medical advice) form.

She was given one more unit of PRBC and urgent MRI was done, which showed focal thinning of the myometrium at the site of previous uterine scar pointing towards dehiscence or complete rupture, as shown in figure 2.

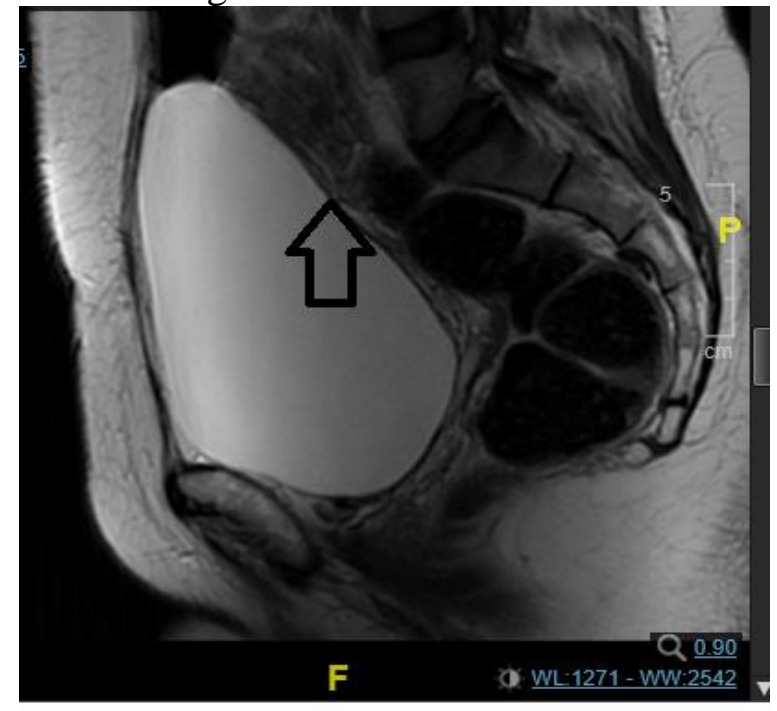

Figure 2: MRI image of the same patient the arrow is showing the ruptured scar

The patient was counselled again and the informed consent was taken for the 
exploratory laparotomy. The need of hysterectomy discussed with the patient but she refused firmly. She agreed after repeated counseling for the laparotomy and the repair of the scar only.

After 6 hours of the collapse she was shifted to operating room (OR) for laparotomy with the arrangement of the 4 units of PRBC and FFP (fresh frozen plasma) respectively. After giving her general anesthesia (as per patient request) the abdomen was opened in Pfannenstiel incision after removing the previous scar under aseptic conditions. Hemo peritoneum was found. Almost $300 \mathrm{cc}$ of the blood was removed from the cavity. The urinary bladder was found adherent with the lower uterine segment which was dissected down. After the dissection, uterus was exteriorized and a $2.5 \mathrm{~cm}$ complete uterine rupture was found on the right side of the previous lower segment transverse scar and dehiscence was noted on the rest of the scar area with the serosa intact, as shown in figure 3.

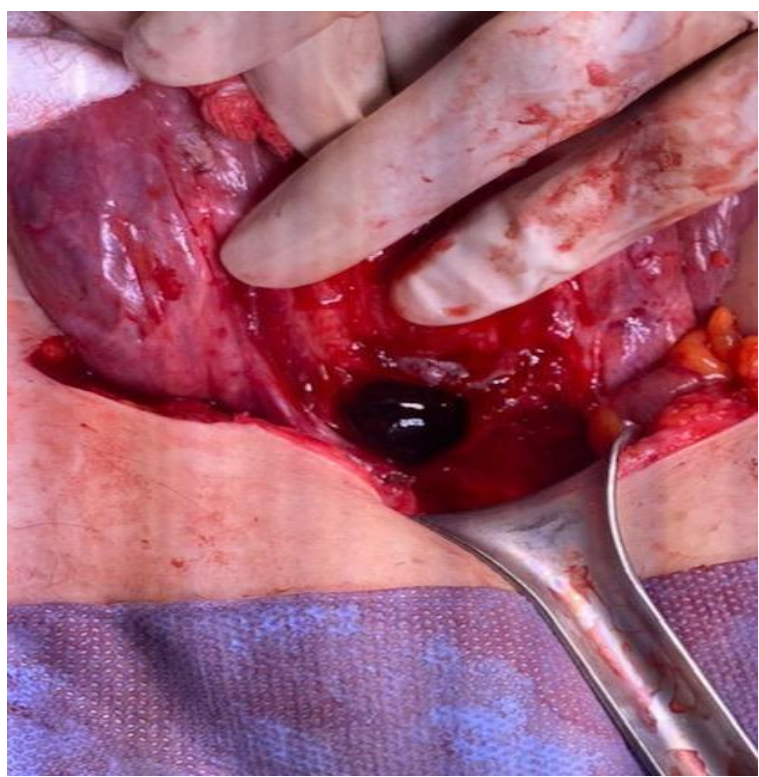

Figure 3: A blood clot is trying to come out of the full thickness uterine defect

The uterine cavity was cleaned and almost 400cc blood clots were removed. After cleaning the cavity thoroughly, the uterine scar was repaired in two layers and the hemostasis was secured. Suction irrigation of the abdominal cavity was done and the abdomen was closed in reverse order after securing the hemostasis. ASD (anti septic dressing) was done. Vagina was cleaned and another $100 \mathrm{cc}$ clots were removed. The estimated amount of the blood loss was 1.2 liters. She received 2 units of PRBC and 2 units of FFP during surgery. Her immediate post op recovery was uneventful and she was shifted back to ICU for continuous monitoring, where she received 4 units of PRBC and 4 units of FFPs, 4 units of platelets and 8 units of cryoprecipitate in the first 36 hours post operative period. On the second post op day she was stable recovering well and was shifted back to the post natal ward. She had uneventful post operative hospital stay.

She did not experience any attack of vaginal bleeding thereafter.

Her HGB was checked on day 2 postoperative, it was $9.6 \mathrm{gram} / \mathrm{dl}$.

The Patient was discharged on the $4^{\text {th }}$ post operative.

She was seen after a week in outpatient department. She was doing well with no bleeding and well healed abdominal wound. The patient was followed up till her first periods after surgery which was found normal and finished after 6 days with mild bleeding.

\section{DISCUSSION}

The chance of rupture of the non gravid uterus is exceptionally rare. Only few cases are reported in the literature. In Iran Geranpayeh and colleagues reported a 63 years old post menopausal women who had previous all normal vertex deliveries and presented with pain and fever, on laparotomy TAH+BSO was done due to perforated pyometra (4).

While Gharabaghi PM and colleagues reported a 40 years old women with previous 2 LSCS on OCP came with bleeding, abdominal pain and vomiting. $\mathrm{He}$ was found to have a longitudinal rupture from the fundus till the cervix, and surprisingly the uterine scar was intact. Her TAH was done with conservation of the ovaries and the reason was again pyometra. (7) In another case in Australia a 71 years old post menopausal women without any 
previous uterine scar presented with sudden severe lower abdominal pain and was having signs of peritonitis. The urgent laparotomy after resuscitation revealed perforated pyometra and she ended up in having hysterectomy as well (6) Herrera and colleagues reported from USA, a 30 years old lady with previous one dilatation and curettage presenting with acute abdomen and on laparotomy, a hemorrhagic uterus was found with a tear extending from the fundus posteriorly to the uterine neck which was repaired(2) In India very recently a rupture from the previous myomectomy scar was reported in a patient with previous 2 LSCS and the myomectomy.(5)

Two more cases were reported back in 1949 and 1952 in one patient she had history of multiple prior surgeries and deep cauterization and the other had fulminant pelvic infection prior to the rupture of the uterus. $(8,9)$

The presentation of all the reported cases is almost the same bleeding, pain, peritonitis and hemorrhagic shock which was noticed in our patient as well.

Our patient had previous 4 LSCS and the rupture was noted at the site of the previous scar which was in contrary to all the previous reported cases earlier, in which site of the rupture was not the scar except in one patient in which the myomectomy scar was found to be the site of the rupture.(5)

Pyometra was the most common cause reported in $71 \%$ of the cases ( $n=5$ out of 7$)$. Unfortunately in $85 \%(n=6 / 7)$ of the patients TAH was the consequence, Only in one patient the uterus was saved.(2) In our patient however the uterus was saved as the patient refused for TAH.

\section{CONCLUSION}

Uterine rupture in the non gravid patient is though extremely rare but should be kept in the differential diagnosis of any female patient presenting with acute abdomen irrespective of the age, parity and the previous surgeries.

\section{ACKNOWLEDGEMENT}

The authors are indebted to the RRT, ICU team and the blood bank for their prompt response and support throughout the Co-management of the patient.

\section{Conflict of Interest: None}

\section{Source of Funding: None}

\section{REFERENCES}

1. Guiliano M, Closset E Therby D, LeGoueff F, Deruelle P, Subtil D. Signs, symptoms and complications of complete and patial uterine ruptures during pregnancy and delivery. Eur J Obstet Gynecol and Reprod bio.2014;179:130-4.

2. Herrera FA, Hassanein AD, Bansal V. Atraumatic spontaneous rupture of the non gravid uterus. J Emerg Trauma Shock. 2011;4(3):439-41.

3. Kieser KE, Baskett TF. A 10 year population based study of uterine rupture. Obstet Gynecol 2002;100:749-53.

4. Geranpayeh L, Fadaei-Araghi M, Shakiba B. Spontaneous uterine perforation due to pyometra presenting as acute abdomen. Infect Dis Obstet Gynecol.2006.

5. Waris NM, Ayyan SM, Rohan KV, Nair SG. A case of spontaneous uterine rupture in a non gravid uterus. Int $\mathbf{J}$ Acad Med. 2020;6:220-3

6. Weng LC, Menon T, Hool G. Spontaneous rupture of the non gravid uterus. BMJ case rep.2013.

7. Gharabaghi PM,Bordbar S, Vazifekhah, Behzad MN. Spontaneous rupture ofthe pyometra in the non pregnant young woman. case Reports in Obstet gynecol, 2017.

8. Frech HC. Spontaneous rupture of the nongravid uterus. South Med J.1949;42:1088.

9. Bornstien FP. Spontaneous rupture of the non-pregnant uterus. Postgrad Med 1952; 12:534-6.

How to cite this article: Najam S, Mohmed SA, Aqeel $S$ et.al. Spontaneous rupture of the non gravid uterus: a case report. Int J Health Sci Res. 2021; 11(11): 108-111. DOI: https://doi.org/10. 52403/ijhsr.20211112 